\title{
Adaptação e Validação da Family Dynamics Measure II para Familiares de Mulheres com Câncer de Mama ${ }^{1}$
}

\author{
Teresa Cristina Martins Leite Imada ${ }^{2}$ \\ Marli Villela Mamede \\ Luiz de Souza \\ Universidade de São Paulo (Ribeirão Preto) \\ Raquel Gabrielli Biffi \\ Centro Universitário Barão de Mauá (Ribeirão Preto)
}

\begin{abstract}
RESUMO - O estudo objetivou adaptar e validar a Family Dynamics Measure II para uso com famílias de mulheres com câncer de mama. O processo incluiu tradução, retrotradução, análise da equivalência semântica pela autora principal do instrumento, análise semântica por amostra da população-alvo, análise de conteúdo por juízes e avaliação das propriedades psicométricas da escala aplicada a 251 familiares de mulheres com câncer de mama. Obteve-se versão adaptada com fidedignidade geral boa $(\alpha=0,90)$; a análise fatorial não confirmou a dimensionalidade teórica do instrumento original; as correlações com a Escala de Ansiedade e Depressão Hospitalar foram invertidas e de baixa a moderada intensidade. A escala foi considerada válida para uso no Brasil. Novos estudos foram sugeridos para fortalecer as evidências.
\end{abstract}

Palavras-chave: escala; dinâmica familiar; oncologia.

\section{Family Dynamics Measure II Adaptation and Validation to Family Members of Women with Breast Cancer}

\begin{abstract}
The study aimed to adapt and validate the Family Dynamics Measure II for use with families of women with breast cancer. The process included translation, back-translation, semantic equivalence analysis by the instrument's main author, semantic analysis per sample of the target population, content validity analysis by judges and evaluation of psychometric properties of the scale applied to 251 relatives of women with breast cancer. It was obtained an adapted version with good reliability $(\alpha=0.90)$; factor analysis did not confirm the theoretical dimensionality of the original instrument; correlations with the Hospital Anxiety and Depression Scale were inverted, and from low to moderate intensity. The scale was considered valid for use in Brazil. Further studies are suggested to strengthen the evidence.
\end{abstract}

Keywords: scale; family dynamics; oncology.

O câncer de mama situa-se como o segundo tipo de câncer mais frequente no mundo e o primeiro entre as mulheres. Os estudos sobre a doença evidenciam que a experiência com o câncer de mama afeta a família como um todo (Fergus \& Gray, 2009; Northouse, Kershaw, Mood \& Schafenacker, 2005; Ozono \& cols., 2005; Raveis \& Pretter, 2005). Conhecer a dinâmica familiar e identificar aspectos da interação entre os membros da família que ficam comprometidos com o surgimento da doença e que prejudicam o ajustamento e a qualidade de vida das mulheres e de suas famílias são etapas iniciais para se desenvolver serviços adequados à população em foco.

1 Este trabalho foi derivado da Tese de Doutorado apresentada ao Departamento Materno-Infantil e de Saúde Pública da Escola de Enfermagem de Ribeirão Preto - USP para obtenção do título de Doutora em Enfermagem em Saúde Pública. Agradecimento especial ao Prof. Dr. Tales Vilela Santeiro por sua colaboração para o artigo.

2 Endereço para correspondência: Rua Acácio de Lima, 678, Chácara Santo Antonio. Franca, SP. CEP 14403-265. Fone: (16) 370112 29; (16) 9121 33 65. Fax: (16) 370254 54. E-mail: h.imada@netsite.com.br.
Considerando a escassez de tempo e de recursos humanos nos serviços de saúde no Brasil, torna-se importante contribuir para que o acesso e a forma de obtenção de informações que possibilitem aos profissionais de saúde o conhecimento sobre o funcionamento familiar após o diagnóstico do câncer de mama sejam facilitados. Uma estratégia que tem se mostrado de grande relevância, com confiabilidade, simplicidade, economia e eficácia, é a utilização de instrumentos de avaliação construídos e adequadamente testados para objetivos pré-definidos.

Não se obteve conhecimento da existência de escalas de medida de dinâmica familiar adaptadas ao uso com famílias de mulheres com câncer de mama no Brasil. Para preencher essa lacuna, tornaram-se o foco desse estudo a adaptação e a validação para a realidade local de um instrumento para o estudo da dinâmica familiar, construído em outro país e cultura. A escala de avaliação de dinâmica familiar eleita para o estudo foi a Family Dynamics Measure II (FDM II) (Lasky \& cols., 1985). Trata-se de um 
instrumento desenvolvido e revisado por um grupo de enfermeiras pesquisadoras norte-americanas, para atender à necessidade de avaliação da dinâmica familiar em situação de saúde e doença, visando o uso clínico e de pesquisa. Essa escala permite o levantamento de dados individuais e familiares que favorecem o reconhecimento de normas familiares e a identificação de perfis das famílias, além de sugerir estratégias de intervenção. A definição de família adotada é a de uma unidade psicossocial composta por duas ou mais pessoas que têm compromissos entre si e moram juntas.

A construção da escala foi baseada na teoria do sistema familiar saudável, proposta por Barnhill (1979), que integrou diversas teorias sobre saúde e doença no funcionamento familiar e definiu oito dimensões básicas, constituídas por polos antagônicos, das quais foram utilizadas seis: (1) individuação-simbiose; (2) mutualidade-isolamento; (3) flexibilidade-rigidez; (4) estabilidade-desorganização; (5) comunicação clara-comunicação confusa ou distorcida; e (6) reciprocidade de papéis-conflito de papéis. As dimensões não utilizadas na escala foram: percepção clara-percepção não clara ou distorcida e limites claros entre as gerações-limites difusos ou perturbados.

A FDM II consiste em um questionário autoaplicável com afirmações positivas e negativas. A primeira versão do instrumento era composta por 62 itens. Essa versão foi revista em 1993 e foram acrescentados mais quatro itens ao instrumento. A FDM II contém 66 itens listados randomicamente em escala tipo Likert de seis pontos, de "concordo plenamente" a "discordo plenamente" (Murtonen, Paunonen, Lehti \& White, 1998).

Para que todos os valores tenham a mesma direção no processo de pontuação é realizada a codificação reversa dos itens negativos. O resultado da escala é obtido por meio do cálculo dos escores de cada uma das dimensões e a avaliação da dinâmica familiar é feita a partir dos resultados parciais. Valores maiores nos itens estão relacionados com dinâmica familiar mais saudável nas dimensões correspondentes.

Vários estudos utilizando a FDM ou FDM II foram realizados com diferentes populações, sendo os mais recentes os de Kanervisto, Paavilainen \& Heikkikä (2007) e White e cols. (2010). Em todos eles, o instrumento mostrou-se satisfatoriamente confiável.

Para verificar a validade e a confiabilidade da FDM II para a avaliação da dinâmica familiar de mulheres brasileiras com câncer de mama, foram propostos para este estudo os seguintes objetivos: (a) realizar a adaptação da FDM II para a língua portuguesa; (b) realizar a análise semântica da versão em português da FDM II; (c) avaliar a validade de conteúdo por juízes; (d) avaliar a validade de construto da escala por meio da análise fatorial; (e) avaliar a validade de construto convergente da FDM II com a Escala de Ansiedade e Depressão Hospitalar (HADS); e (f) avaliar a fidedignidade da versão adaptada da FDM II, verificando a consistência interna dos itens em uma amostra de familiares de mulheres com câncer de mama, no Brasil.

\section{Método}

\section{Participantes}

Participaram da pesquisa, na etapa de adaptação e validação do instrumento, familiares de mulheres com câncer de mama e juízes.

Os critérios para seleção dos participantes foram:

a) Juízes: terem experiência e conhecimento de famílias por meio da atuação profissional ou de pesquisa com essa população;

b) Familiares de mulheres com câncer de mama: ser membro de família na qual houvesse mulher diagnosticada com câncer de mama de há 3 meses até há 5 anos, ou há mais de cinco anos se a mulher ainda estivesse em tratamento; residir no mesmo domicílio da mulher com diagnóstico de câncer de mama; ter 18 anos ou mais; concordar em participar; ter condições para compreensão e realização da tarefa.

$\mathrm{Na}$ análise semântica, a amostra foi composta por nove familiares, principalmente maridos $(55,6 \%)$ e filhas $(11,1 \%)$. As idades variaram entre 26 a 79 anos (Média=47,11; $\mathrm{DP}=19,99$; Mediana=38). Aproximadamente 77,8\% dos participantes eram casados e $55,6 \%$ possuía Ensino Médio completo. $\mathrm{Na}$ análise de conteúdo, participaram nove juízes: três terapeutas de família, dois psicólogos, dois enfermeiros e dois graduandos em Psicologia.

A amostra de participantes da etapa de teste das propriedades psicométricas da FDM II foi composta por 251 familiares de 183 mulheres com câncer de mama, sendo principalmente maridos (39\%), filhas $(29,5 \%)$ e filhos $(17,1 \%)$. A idade dos participantes variou entre 18 e 89 anos, sendo a média de idade igual a 42,51 anos $(\mathrm{DP}=18,3)$ e a mediana igual a 42. A maioria dos participantes era casada $(54,6 \%)$, sendo os demais solteiros $(33,1 \%)$; separados ou divorciados $(5,6 \%)$; viúvos $(3,6 \%)$ e amasiados $(3,2 \%)$. Quanto à escolaridade, a amostra mostrou-se heterogênea, sendo que $80(31,9 \%)$ participantes possuíam Ensino Médio completo, 71 (28,3\%), Ensino Fundamental incompleto, 27 (10,8\%), Ensino Superior completo e seis $(2,4 \%)$ eram analfabetos.

Os Comitês de Ética em Pesquisa da Escola de Enfermagem de Ribeirão Preto - USP e da Fundação Civil Santa Casa de Misericórdia de Franca - Hospital do Câncer de Franca analisaram e aprovaram a realização da pesquisa.

\section{Procedimento}

Para a adaptação e validação da FDM II, utilizou-se uma combinação de orientações metodológicas (Beaton, Bombardier, Guillemin \& Ferraz, 2000; Chwalow, 1995; Maneesriwongul \& Dixon, 2004; Urbina, 2004/2007). A primeira etapa do trabalho consistiu na adaptação da escala, que envolveu os processos de tradução, retrotradução, análise 
semântica pela autora principal da escala, análise semântica por amostra da população-alvo e análise de conteúdo por juízes. Após essa etapa, foi obtida a versão final adaptada em português da FDM II. Na segunda etapa, foram realizados testes das propriedades psicométricas do instrumento, incluindo avaliações da validade por meio da análise fatorial e da análise da validade convergente e da fidedignidade por meio do cálculo do coeficiente alfa de Cronbach. Aqui é priorizada a apresentação dos resultados da pesquisa a partir da análise semântica por amostra da população-alvo.

A versão original da FDM II foi traduzida para o português por três tradutores independentes, dois deles da área de saúde. As versões traduzidas foram discutidas pela equipe de pesquisadores e foi realizada uma síntese das mesmas, sendo obtida a primeira versão em português (VP1). Essa versão foi submetida à retrotradução para o inglês por dois outros tradutores, um deles bilíngue. A equipe discutiu as versões retrotraduzidas e obteve-se a nova versão em inglês (VI2). A VI2 foi encaminhada para a autora principal da escala, que realizou a análise da equivalência semântica dos itens, sugerindo mudanças nos itens $3,21,24,28,32,34$, 49 e 54 . As sugestões da autora foram acatadas, obtendo-se a segunda versão da FDM II em português (VP2).

A seguir, realizou-se a análise semântica para verificar se os itens traduzidos eram compreensíveis. Para isso, foram realizadas entrevistas com nove familiares de mulheres com câncer de mama atendidas em dois serviços de saúde. Seguindo a orientação de Pasquali (2003), os itens foram apresentados um a um e os participantes foram convidados a repetir com suas palavras o conteúdo dos mesmos, a avaliar se o item era compreensível e a sugerir melhorias, caso eles considerassem o item de difícil entendimento. Os comentários dos participantes a respeito de cada item foram registrados. Houve dificuldade de compreensão em 17 itens $(1,3,4,5,9,10,14,22,29,32,34,37,43,44,52,58$ e 63$)$ e foram dadas sugestões para a modificação de 10 itens (15, $16,20,29,31,32,40,47,50,54)$. Foi feita uma revisão do instrumento e os itens $1,5,10,16,22,29,31,32,40,43,47$, 54,58 e 63 foram modificados, dando origem à VP3.

A versão resultante desse processo foi submetida à análise do conteúdo por juízes. Os juízes receberam uma lista impressa com as definições teóricas das dimensões avaliadas pelo instrumento e foram convidados a categorizar os itens da escala nas dimensões da dinâmica familiar correspondentes, assinalando as categorias numa tabela. Avaliou-se a frequência de concordância com que os juízes procediam à categorização de cada item de forma semelhante à proposta pelas autoras. A concordância da classificação dos itens nas dimensões da dinâmica familiar entre os juízes e as autoras da escala variou de $0 \mathrm{a}$ $100 \%$. Em 19 itens $(1,8,13,14,24,25,30,31,36,43,44,47$, 51 ) houve $100 \%$ de concordância entre os juízes e as autoras. Os itens nos quais houve total discordância entre os juízes e as autoras foram o 23 e o 29. Foi realizada nova revisão dos itens do instrumento, sendo os itens 23, 26, 27, 46 e 61 corrigidos do ponto de vista ortográfico. Assim, obteve-se a versão final adaptada da FDM II (Apêndice A). Essa versão foi submetida ao teste de suas propriedades psicométricas, o que constituiu a segunda etapa do processo de avaliação do instrumento.
Para verificar se os itens da escala representavam adequadamente as dimensões da dinâmica familiar de acordo com o proposto pela teoria, foi realizada a análise da validade do construto. A validade de construto foi avaliada pela análise fatorial e pela análise da validade convergente da FDM II com a HADS (Botega, Bio, Zomignani, Garcia Jr. \& Pereira, 1995). A análise da fidedignidade ou, em outras palavras, da estabilidade da medida, foi realizada por meio do cálculo do coeficiente alfa de Cronbach. Para essas análises foram aplicadas as versões adaptadas da FDM II, da HADS e uma ficha de identificação sociodemográfica do participante e da mulher em uma amostra de familiares de mulheres com câncer de mama.

Mulheres com câncer de mama usuárias dos serviços do REMA - Núcleo de Ensino, Pesquisa e Assistência na Reabilitação de Mastectomizadas da Escola de Enfermagem de Ribeirão Preto da Universidade de São Paulo e do Hospital do Câncer de Franca foram contatadas, sendo-lhes explicados os objetivos e a forma de participação na pesquisa. Com o seu consentimento, as mulheres ou a primeira pesquisadora convidaram os familiares para participarem. As entrevistas foram agendadas previamente após confirmação do interesse e da disponibilidade dos familiares em participar e realizadas em horários e locais convenientes para os participantes (no domicílio ou nas dependências dos serviços de saúde).

As entrevistas tiveram como objetivo a aplicação dos instrumentos. Houve nova explicação aos participantes dos objetivos e procedimentos da pesquisa e foram dadas informações sobre os direitos dos participantes, conforme normas que regulamentam as pesquisas com seres humanos, contidas na Resolução 196/96 do Ministério da Saúde/CONEP. Após a assinatura dos termos de consentimento pelo familiar e pela mulher com câncer de mama, foram aplicados os demais instrumentos.

As escalas utilizadas são autoaplicáveis; porém, optou-se pela aplicação pela primeira pesquisadora para assegurar que nenhum item deixasse de ser respondido.

\section{Resultados}

A Tabela 1 apresenta os resultados da estatística descritiva da versão adaptada da FDM II aplicada aos familiares de mulheres com câncer de mama.

As médias e as medianas de todas as dimensões da versão adaptada da FDM II apresentaram valores acima do valor médio do intervalo possível para cada uma delas. Todos os itens positivos, exceto o item 10, tiveram médias acima do valor médio do intervalo possível para os itens, indicando maior concordância com eles, conforme o esperado. Da mesma forma, a maioria dos itens negativos teve média abaixo do valor médio do intervalo possível para o item, indicando maior discordância deles. Os itens negativos que tiveram médias acima dos valores médios dos intervalos possíveis para os itens foram: $8,11,15,38,39$ e 60 .

$\mathrm{Na}$ avaliação da fidedignidade, medida pelo coeficiente alfa de Cronbach, obteve-se $\alpha=0,90$, o que indica que a es- 
Tabela 1. Estatística descritiva da versão adaptada da FDM II, médias das dimensões, desvios padrão, medianas e intervalos de escores possíveis e obtidos $(\mathrm{n}=251)$, usando codificação reversa dos itens negativos.

\begin{tabular}{|c|c|c|c|c|}
\hline Dimensões & $\begin{array}{l}\text { Intervalo } \\
\text { possível }\end{array}$ & $\begin{array}{l}\text { Intervalo } \\
\text { obtido }\end{array}$ & Mediana & Média (DP) \\
\hline 1. Individuação/Simbiose & $13-78$ & $36-69$ & 53 & $52,17(6,25)$ \\
\hline 2. Mutualidade/Isolamento & $11-66$ & $24-66$ & 53 & $52,66(6,62)$ \\
\hline 3. Flexibilidade/Rigidez & $10-60$ & $23-56$ & 38 & $38,3(5,41)$ \\
\hline 4. Estabilidade/Desorganização & $9-54$ & $22-52$ & 42 & $40,88(5,37)$ \\
\hline $\begin{array}{l}\text { 5. Comunicação clara/Comunicação } \\
\text { confusa ou distorcida }\end{array}$ & $11-66$ & $26-65$ & 51 & $49,24(7,35)$ \\
\hline $\begin{array}{l}\text { 6. Reciprocidade de papéis/ } \\
\text { Conflito de papéis }\end{array}$ & $12-72$ & $24-66$ & 53 & $51,26(6,82)$ \\
\hline
\end{tabular}

cala possui um grau de precisão elevado. Considerando-se as dimensões definidas pelas autoras, os coeficientes alfa de Cronbach foram: (1) individuação/simbiose=0,48; (2) mutualidade/isolamento $=0,82$; (3) flexibilidade/ rigide $z=0,55 ;$ (4) estabilidade/desorganização=0,69; (5) comunicação clara/ comunicação confusa ou distorcida $=0,79$; e (6) reciprocidade de papéis/conflito de papéis $=0,64$. Ou seja, nas dimensões 2 e 5 , a consistência interna dos itens foi muito boa. Na dimensão 4 , a consistência interna dos itens foi razoável. Nas dimensões 1, 3 e 6, a consistência interna dos itens foi insatisfatória, conforme critérios definidos por Pasquali (2003).

$\mathrm{Na}$ Tabela 2 encontram-se os coeficientes alfa de Cronbach geral e para as dimensões da escala, comparados com os obtidos pelas autoras em três amostras pesquisadas.

A comparação entre os coeficientes alfa para cada dimensão com os obtidos pelas autoras indica que, apesar de a fidedignidade ter sido baixa ou moderada em alguns domínios, os resultados foram próximos nas dimensões 1 , 2, 3, 4 e 5. Houve maior discrepância de coeficientes na dimensão 6. É importante mencionar que os resultados das autoras referem-se aos testes realizados com a escala em sua primeira versão. Em todas as dimensões, a exclusão de itens não altera significativamente os valores de alfa, não havendo justificativa para a retirada de nenhum item do instrumento.

A análise de fatores revelou 20 dimensões do instrumento de 66 itens com autovalores (eigenvalues) maiores do que 1 , explicando $65,2 \%$ da variância total. Os seis primeiros construtos explicam $36,7 \%$ da variância total, com autovalores iguais ou maiores que 2,0. Na amostra testada, a análise fatorial revelou que não houve concordância dos fatores obtidos com a proposta teórica das autoras sobre a dimensionalidade da escala. Os itens 9, 16, 52, 60 e 66 tiveram cargas fatoriais menores que 0,30 , apresentando comunalidades iguais a 0,12 ; 0,$22 ; 0,08 ; 0,09$ e 0,14 , respectivamente, as quais são con-

Tabela 2. Comparação dos coeficientes alfa de Cronbach geral e por dimensões da versão adaptada da escala FDM II com os obtidos pelas autoras em três amostras usando a FDM.

\begin{tabular}{ccccc} 
& Versão & \multicolumn{3}{c}{ Populações investigadas } \\
$\alpha$ de Cronbach & adaptada & Normal & Risk & Illness
\end{tabular}

(66 itens)

(55 itens)

(55 itens)

(55 itens)

Geral

1. Individuação/Simbiose

2. Mutualidade/Isolamento

3. Flexibilidade/Rigidez

4. Estabilidade/Desorganização

5. Comunicação clara/Comunicação confusa ou distorcida

6. Reciprocidade de papéis/

Conflito de papéis
0,90

0,48

0,82

0,48

0,60

0,46

0,55

0,88

0,84

0,88

0,69

0,64

0,53

0,61

0,85

0,72

0,69

0,79

0,88

0,83

0,83

0,64

0,76

0,77

0,79 
sideradas baixas (próximas de zero). Retirando esses itens, observa-se que o instrumento melhora o seu desempenho na porcentagem de variância $(36,7 \%$ x 39,4\%) e na dimensão $1(0,48 \times 0,53)$, e piora na dimensão $6(0,64$ x 0,62). Como há mais ganho do que perda, sugere-se a adaptação do instrumento sem tais itens.

Os valores obtidos nas correlações entre as medidas de ansiedade da HADS e as medidas das dimensões 4, 5 e 6 da versão adaptada da FDM II foram de sentido inverso e de intensidade moderada $(\mathrm{r}=-0,38, \mathrm{p}<0,001 ; \mathrm{r}=-0,34, \mathrm{p}<0,001$; $\mathrm{r}=-0,30, \mathrm{p}<0,001$, respectivamente), conforme critérios definidos por Andresen (2000). As correlações entre as medidas de ansiedade da HADS com as medidas das dimensões 1, 2 e 3 da FDM II foram fracas. Nas correlações entre as medidas de depressão e as medidas das dimensões 2, 4, 5 e 6 da versão adaptada da FDM II, obtiveram-se correlações também de sentido inverso e de intensidade moderada $(\mathrm{r}=-0,34, \mathrm{p}<0,001$, $\mathrm{r}=-0,35, \mathrm{p}<0,001, \mathrm{r}=-0,34, \mathrm{p}<0,001, \mathrm{r}=-0,35, \mathrm{p}<0,001$, respectivamente). Por outro lado, as correlações das medidas de depressão da HADS com as medidas das dimensões 1 e 3 da FDM II foram também de sentido inverso, porém fracas. As medidas das dimensões 1 e 3 não apresentaram correlação suficiente com as medidas de ansiedade e de depressão da HADS.

\section{Discussão}

Foram realizadas várias etapas para a adaptação transcultural da FDM II e a cada etapa o instrumento foi revisto e algumas modificações foram feitas, alcançando-se uma versão adaptada da FDM II, considerada adequada para ser submetida ao teste de suas propriedades psicométricas. $\mathrm{Na}$ análise semântica por amostra da população-alvo, o item 1 teve maior frequência de dúvidas, provavelmente por ser o primeiro do instrumento, apesar da tarefa ter sido cuidadosamente explicada a cada participante. A maior frequência de dúvidas em itens da dimensão 1 (individuação/simbiose) em comparação com as demais dimensões talvez se explique por essa dimensão se referir a aspectos bastante subjetivos do funcionamento familiar.

$\mathrm{Na}$ análise de conteúdo por juízes, os resultados obtidos indicaram que em mais de um quarto dos itens houve concordância de $100 \%$ entre os juízes e as autoras do instrumento original quanto à correspondência entre os itens e as dimensões. Nas dimensões 1, 2, 3 e 5, a média de concordância por dimensão superou $70 \%$, enquanto nas dimensões 4 e 6 essas médias foram de aproximadamente $63 \%$. Esses valores sugerem que o conteúdo teórico do instrumento traduzido está satisfatoriamente adequado para avaliar o funcionamento de famílias. Nos itens 23 e 29 houve total discordância entre os juízes e as autoras quanto à dimensionalidade dos itens. Levanta-se a hipótese de haver uma diferença cultural na forma de entender esses itens. Para as autoras, o relacionamento com os outros tem um sentido mais pragmático, de papéis desempenhados em conjunto (reciprocidade de papéis), enquanto para a maioria dos juízes foi entendido mais como uma proximidade dos membros da família (mutualidade). As autoras consideram o item 29 um representante de rigidez e os juízes consideraram-no mais como um representante da estabilidade familiar. O grau de fixidez indicado pelo item pode não ter ficado claro na sua tradução para o português.

Nesse estudo, a versão adaptada da FDM II foi aplicada em 251 familiares, havendo um predomínio da participação de esposos e filhas, o que confirma as observações de Bernard e Guarnaccia (2003), Grunfeld e cols. (2004) e Northouse e cols. (2005) de que esses membros da família muitas vezes assumem o papel de cuidadores da mulher com câncer de mama, acompanhando-a aos serviços de saúde e atuando como intermediários e porta-vozes em relação aos demais membros do grupo familiar. A análise estatística descritiva das respostas dos participantes ao instrumento revelou que as médias das dimensões ficaram todas acima do valor médio do intervalo possível para cada uma delas. Sabendo-se que escores mais altos indicam famílias mais saudáveis, esses dados podem ser interpretados como sinal de predomínio de saúde no funcionamento familiar da amostra pesquisada.

A dimensão 2 (mutualidade/isolamento) teve a média de resposta mais elevada da escala. Essa mesma dimensão teve também a mais alta média de concordância dos juízes com as autoras na análise de conteúdo e foi a dimensão na qual a maioria dos itens teve carga fatorial acima de 0,50 num mesmo fator, o que indica a validade dessa dimensão do instrumento.

Um item considerado positivo teve frequência de resposta de discordância acima do esperado e cinco itens considerados negativos tiveram resposta de discordância abaixo do esperado. A frequência de respostas acima ou abaixo da qual era esperada, conforme o tipo de item (positivo ou negativo), sugere que pode haver diferenças culturais entre o país de origem do instrumento e o país para o qual o instrumento está sendo adaptado. Se, no país de origem do instrumento, tomar decisões por conta própria seja um sinal de autonomia e independência (individuação), e depender da opinião de outros membros da família seja visto de forma negativa, para a amostra pesquisada, a interdependência dos membros da família é algo valorizado, pelo menos nesse momento da vida em que uma doença está sendo enfrentada. A análise desses itens nas demais etapas da pesquisa mostra que a diferença de frequência de respostas em relação ao esperado provavelmente está mesmo relacionada com significados culturais atribuídos aos mesmos.

$\mathrm{Na}$ análise fatorial, a distribuição das cargas fatoriais dos itens por dimensão revela que não houve concordância dos fatores obtidos com a dimensionalidade do instrumento proposta teoricamente pelas autoras. Algumas hipóteses são levantadas para explicar esse resultado. $\mathrm{Na}$ análise fatorial do instrumento original, em sua primeira versão (FDM), as autoras também não confirmaram a dimensionalidade teórica do instrumento. Portanto, esse resultado seria indicativo de uma característica do próprio instrumento. $\mathrm{O}$ construto "dinâmica familiar" é complexo e envolve um conjunto de dimensões que se inter-relacionam, sendo difícil distingui-los claramente. Segundo Floyd e Widaman (1995), construtos psicológicos compostos por múltiplas facetas correlacionadas muitas vezes não se conformam aos modelos que requerem que essas facetas apresentem cargas em um único fator. Esse pode ser o caso do construto investigado pela FDM II. Além disso, o número elevado de itens por dimensão pode ser mais um dos motivos para a dificuldade em confirmar 
a dimensionalidade teórica proposta pelas autoras. Floyd e Widaman (1995) apontam que questionários moderadamente longos, com mais de cinco itens por dimensão, tornam mais difícil a confirmação da estrutura fatorial. Ao retirar os itens que tiveram cargas fatoriais menores que 0,30 na análise fatorial $(9,16,52,60$ e 66), verificou-se haver mais ganho do que perda com a retirada desses itens; portanto, sugere-se a adaptação do instrumento sem eles.

Nas correlações entre as medidas de ansiedade da HADS com as medidas das dimensões da FDM II, verificou-se que a ansiedade está associada com o nível de organização das relações familiares, bem como com as trocas comunicativas e instrumentais entre os membros da família. Quanto maior a ansiedade, mais desorganizada a família, mais distorcida e confusa a comunicação entre seus membros e mais conflitos ocorrem entre os papéis desempenhados por eles. Por outro lado, a ansiedade não se relaciona com a autonomia, independência e proximidade dos indivíduos dentro da família, nem tampouco com a possibilidade de modificações dos padrões familiares.

Com relação às correlações entre a medida de depressão da HADS com as medidas das dimensões da versão adaptada da FDM II, constatou-se que maiores níveis de depressão estão relacionados com mais isolamento, maior desorganização familiar, comunicação mais confusa ou distorcida e mais conflitos de papéis. Porém, não há interferência do nível de depressão sobre a autonomia dos membros da família ou sua flexibilidade.

$\mathrm{Na}$ avaliação da fidedignidade, constatou-se que o instrumento em estudo apresenta uma ótima fidedignidade. Analisando os coeficientes alfa de Cronbach obtidos para cada dimensão, verificou-se que nas dimensões 2 e 5 os resultados obtidos foram muito bons, indicando a precisão dessas dimensões do instrumento. Para a dimensão 4, o resultado pode ser considerado muito próximo do satisfatório. Para as demais dimensões (1, 3 e 4), os coeficientes alfa de Cronbach obtidos foram baixos.

Comparando os resultados deste estudo com os obtidos pelas autoras na adaptação do instrumento original (Lasky \& cols., 1985), verifica-se semelhança entre eles, assim como também se observa semelhança entre os coeficientes alfa de Cronbach deste estudo e aqueles obtidos em outras pesquisas que utilizaram a FDM II (Kanervisto \& cols., 2007; Tammentie, Tarkka, Astedt-Kurki, Paavilainen \& Laippala, 2004; White \& cols., 2003). Com base nessas comparações, confirma-se a fidedignidade da versão adaptada da FDM II para o português.

\section{Considerações Finais}

Os resultados da adaptação e validação da FDM II em amostra de familiares de mulheres com câncer de mama no Brasil possibilitou concluir que a versão em português da FDM II, obtida após os processos de tradução, retrotradução, análise pela autora principal, análise semântica e análise de conteúdo por juízes mostrou-se adaptada para uso com familiares de mulheres com câncer de mama no pais. Na análise fatorial, a dimensão que obteve cargas fatoriais mais elevadas em um mesmo fator foi a 2 (mutualidade/isolamento), não se confirmando a dimensionalidade do instrumento como um todo. A análise da validade de construto convergente resultou em correlações inversas de moderada a baixa intensidade entre as medidas de ansiedade e depressão e as medidas das dimensões da FDM II. As dimensões 4 (estabilidade/desorganização), 5 (comunicação clara/comunicação confusa ou distorcida) e 6 (reciprocidade de papéis/conflito de papéis) estão relacionadas inversamente tanto com ansiedade quanto com depressão. O valor do coeficiente alfa de Cronbach indicou a fidedignidade do instrumento. Os coeficientes alfa para as dimensões 1 (individuação/simbiose), 3 (flexibilidade/rigidez) e 4 (estabilidade/desorganização) foram baixos, indicando a necessidade de novas pesquisas em amostras maiores e diferentes para se verificar se alguns itens precisam ser revistos.

Atualmente, a atenção às famílias tem se mostrado uma prioridade. Instrumentos de avaliação que sirvam de orientação para os profissionais dirigirem sua intervenção para as verdadeiras necessidades da família são muito úteis. $\mathrm{Na}$ ausência de instrumentos nacionais, a adaptação de um instrumento estrangeiro é uma alternativa válida. O processo de adaptação e validação não se encerra após um único estudo. As evidências de validade e fidedignidade devem ser acumuladas para fortalecer a confiança na utilidade do instrumento. Por ora, considera-se que a versão adaptada da FDM II pode ser utilizada no Brasil para avaliar o funcionamento familiar de mulheres com câncer de mama, e estudos com outras amostras são indicados para se testar a possibilidade de generalizar o uso do instrumento para outras populações.

\section{Referências}

Andresen, E. M. (2000). Criteria for assessing the tools of disability outcomes. Archives of Physical Medicine and Reabilitation, 81, 15-20.

Barnhill, L. R. (1979). Healthy family systems. The Family Coordinator, 22, 94-100.

Beaton, D. E., Bombardier, C., Guillemin, F., \& Ferraz, M. C. (2000). Guidelines for the process of cross-cultural adaptation of self-report measures. Spine, 25, 3186-3191.

Bernard, L. L., \& Guarnaccia, C. A. (2003). Two models of caregiver strain and bereavement adjustment: A comparison of husbands and daughters caregivers of breast cancer hospice patients. The Gerontologist, 43, 808-816.

Botega, N. J., Bio, M. R., Zomignani, M. A., Garcia Jr, C., \& Pereira, W. A. B. (1995). Transtornos do humor em enfermaria de clínica médica e validação de escala de medida (HAD) de ansiedade e depressão. Revista de Saúde Pública, 29, 355-363.

Chwalow, A. J. (1995). Cross-cultural validation of existing quality of life scales. Patient Education and Counseling, 26, 313318.

Fergus, K. D., \& Gray, R. E. (2009). Relationship vulnerabilities during breast cancer: Patient and partner perspectives. PsychoOncology, 18, 1311-1322. Retirado em 12/08/2010, de http:// www3.interscience.wiley.com/cgi-bin/fulltext/122302400/ PDFSTART.

Floyd, F. J., \& Widaman, K. F. (1995). Factor analysis in the development and refinement of clinical assessment instruments. Psychological Assessment Issue, 7, 286-299. 
Grunfeld, E., Coyle, D., Whelan, T., Clinch, J., Reyno, L., Earle, C. C., Willan, A., Viola, R., Coristine, M., Janz, T., \& Glossop, R. (2004). Family caregiver burden: Results of a longitudinal study of breast cancer patients and their principal caregivers. Canadian Medical Association Journal, 170, 1795-1801.

Kanervisto, M., Paavilainen, E., \& Heikkilä, J. (2007). Family dynamics in families of severe COPD patients. Journal Clinical Nursing, 16, 1498-1505.

Lasky, P., Buckwalter, K. C., Whall, A., Lederman, R., Speer, J., McLane, A., King, J. M., \& White, M. A. (1985). Developing an instrument for the assessment of family dynamics. Western Journal of Nursing Research, 7, 40-57.

Maneesriwongul, W., \& Dixon, J. K. (2004). Instrument translation process: A method review. Journal of Advanced Nursing, 48, 175-186.

Murtonen, I., Paunonen, M., Lehti, K., \& White, M. (1998). Family dynamics of families with cancer in Finland. Cancer Nursing, 21, 252-258.

Northouse, L., Kershaw, T., Mood, D., \& Schafenacker, A. (2005). Effects of a family intervention on the quality of life of women with recurrent breast cancer and their family caregivers. Psycho-Oncology, 14, 478-491.

Ozono, S., Saeki, T., Inoue, S., Mantani, T., Okamura, H., \& Yamawaki, S. (2005). Family functioning and psychological distress among Japanese breast cancer patients and families. Supportive Care in Cancer, 13, 1044-1050.
Pasquali, L. (2003). Psicometria: teoria dos testes na psicologia e na educação. Petrópolis: Vozes.

Raveis, V. H., \& Pretter, S. (2005). Existential plight of adult daughters following their mother's breast cancer diagnosis. PsychoOncology, 14, 49-60.

Tammentie, T., Tarkka, M. T., Astedt-Kurki, P., Paavilainen, E., \& Laippala, P. (2004). Family dynamics and postnatal depression. Journal of Psychiatric and Mental Health Nursing, 11, 141-149.

Urbina, S. (2007). Fundamentos da testagem psicológica (C. Dornelles, Trad.). Porto Alegre: Artmed (Trabalho original publicado em 2004).

White, M. A., Elder, J. H., Paavilainem, E., Joronen, K., Helgadóttir, H. L., \& Seidl, A. (2010). Family dynamics in the United States, Finland and Iceland. Scandinavian Journal of Caring Sciences, 24, 84-93.
Recebido em 24.06 .08

Primeira decisão editorial em 23.06.10

Versão final em 20.09.10

Aceito em 20.09.10 


\section{Apêndice A}

Itens da versão final adaptada da FDM II.

\begin{tabular}{|c|c|c|}
\hline Itens: Em minha família... & Itens: Em minha família... & Itens: Em minha família... \\
\hline $\begin{array}{l}\text { 1. As atividades do dia-a-dia podem ser } \\
\text { mudadas }\end{array}$ & $\begin{array}{l}\text { 23. Nosso relacionamento uns com os } \\
\text { outros é bom }\end{array}$ & 45. Eu sou um "solitário" \\
\hline $\begin{array}{l}\text { 2. Eu acho que conversamos sobre as } \\
\text { coisas importantes }\end{array}$ & 24. Para mim sobram os piores trabalhos & $\begin{array}{l}\text { 46. Eu sei quando os membros estão } \\
\text { aborrecidos pela forma como eles apa- } \\
\text { rentam }\end{array}$ \\
\hline 3. Eu cuido dos outros & $\begin{array}{l}\text { 25. Algumas pessoas dizem uma coisa e } \\
\text { querem dizer outra }\end{array}$ & $\begin{array}{l}\text { 47. Quando há um mal entendido, } \\
\text { nós conversamos sobre o assunto até } \\
\text { esclarecê-lo }\end{array}$ \\
\hline $\begin{array}{l}\text { 4. Eu tenho um lugar para as minhas } \\
\text { próprias coisas }\end{array}$ & $\begin{array}{l}\text { 26. Visitas de nossos amigos não nos } \\
\text { aborrecem }\end{array}$ & $\begin{array}{l}\text { 48. Falar sobre meus problemas confunde } \\
\text { mais as coisas }\end{array}$ \\
\hline $\begin{array}{l}\text { 5. Nós concordamos com a maneira pela } \\
\text { qual as tarefas são divididas }\end{array}$ & $\begin{array}{l}\text { 27. Eu não sei como o dinheiro da família } \\
\text { será gasto }\end{array}$ & $\begin{array}{l}\text { 49. Parece que alguma coisa está sempre } \\
\text { dando errado }\end{array}$ \\
\hline $\begin{array}{l}\text { 6. Eu penso que conversar não me leva a } \\
\text { lugar nenhum }\end{array}$ & $\begin{array}{l}\text { 28. Eles querem que eu goste da mesma } \\
\text { comida }\end{array}$ & $\begin{array}{l}\text { 50. Eu tenho um lugar onde eu posso ficar } \\
\text { sozinho }\end{array}$ \\
\hline $\begin{array}{l}\text { 7. Eu não consigo ajuda o suficiente com } \\
\text { o trabalho em casa }\end{array}$ & 29. Eu me limito às minhas rotinas diárias & 51. Não posso quebrar as regras \\
\hline 8. Eu raramente mudo as minhas rotinas & $\begin{array}{l}\text { 30. Existe alguém que se preocupa } \\
\text { comigo }\end{array}$ & $\begin{array}{l}\text { 52. Eu deixo que os outros decidam por } \\
\text { mim }\end{array}$ \\
\hline $\begin{array}{l}\text { 9. Eu sei o que posso esperar de um dia } \\
\text { para o outro }\end{array}$ & $\begin{array}{l}\text { 31. É permitido que eu tenha minhas } \\
\text { próprias opiniões }\end{array}$ & 53. É difícil mudar as regras \\
\hline $\begin{array}{l}\text { 10. Eu tomo minhas decisões por conta } \\
\text { própria }\end{array}$ & 32. Eu tenho intimidade & 54. É difícil dizer o que eu quero falar \\
\hline $\begin{array}{l}\text { 11. A maneira correta de fazer as coisas é } \\
\text { importante }\end{array}$ & $\begin{array}{l}\text { 33. Quando eu falo, alguém ouve o que } \\
\text { estou dizendo }\end{array}$ & 55. Ninguém se preocupa comigo \\
\hline $\begin{array}{l}\text { 12. Eu acho justa a minha parte do traba- } \\
\text { lho doméstico }\end{array}$ & 34. Eu me protejo por conta própria & 56. Eu não me sinto próximo de ninguém \\
\hline $\begin{array}{l}\text { 13. Uma vez que se toma uma decisão, é } \\
\text { difícil mudar }\end{array}$ & $\begin{array}{l}\text { 35. Quando as coisas dão erradas, nós } \\
\text { tentamos diferentes maneiras de lidar } \\
\text { com elas }\end{array}$ & $\begin{array}{l}\text { 57. Eu tenho coisas que pertencem a mim } \\
\text { mesmo }\end{array}$ \\
\hline $\begin{array}{l}\text { 14. Eu percebo uma sensação de acon- } \\
\text { chego }\end{array}$ & $\begin{array}{l}\text { 36. Nós fazemos perguntas quando não } \\
\text { entendemos uns aos outros }\end{array}$ & $\begin{array}{l}\text { 58. Nós sabemos como entrar em contato } \\
\text { com os membros da família se necessário }\end{array}$ \\
\hline $\begin{array}{l}\text { 15. Eu guardo os sentimentos comigo } \\
\text { mesmo }\end{array}$ & $\begin{array}{l}\text { 37. Preciso lembrar os outros para faze- } \\
\text { rem suas tarefas }\end{array}$ & $\begin{array}{l}\text { 59. Estou satisfeito com a forma pela qual } \\
\text { o trabalho é feito }\end{array}$ \\
\hline $\begin{array}{l}\text { 16. Eu deixo os outros saberem o que eu } \\
\text { quero }\end{array}$ & $\begin{array}{l}\text { 38. Eu não faço coisas a menos que } \\
\text { alguém concorde }\end{array}$ & 60. Eu penso que somos todos iguais \\
\hline $\begin{array}{l}\text { 17. Eu sei que nós podemos resolver as } \\
\text { coisas quando elas estão erradas }\end{array}$ & $\begin{array}{l}\text { 39. É importante que todos nós pensemos } \\
\text { da mesma maneira }\end{array}$ & 61. Tudo desmorona quando há problemas \\
\hline 18. Eu me sinto excluído & $\begin{array}{l}\text { 40. Os outros esperam que eu me } \\
\text { comporte de maneiras com as quais eu } \\
\text { discordo }\end{array}$ & 62. Nós evitamos falar sobre problemas \\
\hline $\begin{array}{l}\text { 19. Está tudo bem se cada um fizer as } \\
\text { tarefas do seu jeito }\end{array}$ & 41. É fácil mudar os planos & $\begin{array}{l}\text { 63. Eu não sei o que esperar de um dia } \\
\text { para o outro }\end{array}$ \\
\hline $\begin{array}{l}\text { 20. Quando eu me sinto triste, alguém me } \\
\text { consola }\end{array}$ & $\begin{array}{l}\text { 42. Eu faço mais tarefas além das que } \\
\text { foram repartidas comigo }\end{array}$ & $\begin{array}{l}\text { 64. É importante saber onde os membros } \\
\text { da família estão }\end{array}$ \\
\hline $\begin{array}{l}\text { 21. Eu penso que nós evitamos falar de } \\
\text { assuntos que a família precisaria discutir }\end{array}$ & 43. Eu tenho a sensação de união & 65. Eu tenho opinião própria \\
\hline $\begin{array}{l}\text { 22. Os outros oferecem ajuda nas minhas } \\
\text { tarefas }\end{array}$ & $\begin{array}{l}\text { 44. Eu sei o que posso esperar dos outros } \\
\text { membros }\end{array}$ & $\begin{array}{l}\text { 66. Eu não gosto do trabalho que eu tenho } \\
\text { que fazer }\end{array}$ \\
\hline
\end{tabular}

